\title{
Deutscher Verein für öffentliche und private Fürsorge (Hrsg.) (2019). Das Bundesteilhabegesetz zwischen Anspruch und Umsetzung
}

\author{
Berlin: Archiv für Wissenschaft und Praxis der sozialen Arbeit (Heft 1/2019), \\ ISBN 978-3-7841-3123-8, 96 S., 14,50€
}

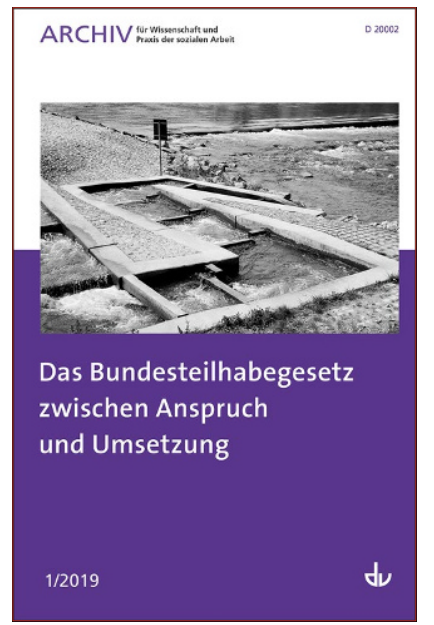

In der vom Deutschen Verein veröffentlichten Publikation geht es um unterschiedliche Themenbereiche, die die Frage der Umsetzung des Bundesteilhabegesetzes für Menschen mit Behinderung betreffen. Dabei geht es sowohl um die Lebenssituation als auch die Beurteilung der neuen Entwicklung in diesem Bereich aus der Sicht der Betroffenen. Es geht aber auch um die Frage nach den Instrumenten der Bedarfsermittlung, nach der Teilhabeplanung und nach der Angemessenheit der Leistungen.

Der Beauftragte der Bundesregierung für die Belange von Menschen mit Behinderung, Jürgen Dusel, begrüßt zwar die Bereitschaft der Verantwortlichen, das deutsche Recht im Sinne der UN-Behindertenkonvention zu erweitern, verkennt aber die hiermit verbundenen Probleme keineswegs. Der für Deutschland typische weitreichende Ausbau der Sonderbereiche hat sich bis heute nicht zugunsten tatsächlicher Fortschritte im Bereich Integration und Inklusion verändert. Auch die zunehmend dominanter werdende Marktorientierung und der immense bürokratische Aufwand, der mit der konkreten Umsetzung von Teilhabe verbunden ist, haben sich bisher nicht eindämmen lassen.

Annett Löwe und Florian Steinmüller beschreiben die Umsetzung des Bundesteilhabegesetzes in den Bundesländern und erläutern detailliert die Bereiche, auf die es
Auswirkungen hat. Natalie Taubert resümiert die ersten Erfahrungen, die Betroffene mit dem neuen Gesetz gemacht haben. Obwohl noch viel zu tun ist, um auch die Bereiche Schule und Ausbildungsmarkt für Menschen mit hohem Unterstützungsbedarf zu öffnen, stellt sie mit Befriedigung fest, dass Begriffe wie Inklusion und Barrierefreiheit inzwischen für eine relativ breite Bevölkerung geläufige und akzeptierte Begriffe sind.

Heike Engel und Thomas Schmidt-Schäfer erläutern die personenzentrierten Instrumente der Bedarfsermittlung und die Voraussetzungen, die diese erfüllen müssen, um ihrem Zweck angemessener Unterstützungsstrategien gerecht zu werden.

Weitere thematische Aspekte, die in der Publikation berücksichtigt werden, sind die Teilhabeplanung im Rahmen des trägerübergreifenden Reha-Prozesses (Marcus Schian/Bernd Giraud) und das Peer Councelling im Rahmen der Teilhabeberatung (Gisela Hermen/Matthias Rösch).

Trotz der Fortschritte, die das neue Gesetz in einigen Bereichen zu verzeichnen hat, kommt Albrecht Rohrmann, ausgewiesener Inklusionsexperte, zu der Schlussfolgerung: „Es lässt sich festhalten, dass das Bundesteilhabegesetz den Herausforderungen des durch die UN-Behindertenkonvention profilierten Paradigmenwechsels nur sehr bedingt gerecht wird. Die aktive Umsetzung der Konvention durch geeignete, wirksame und verbindliche Maßnahmen ist in zentralen Bereichen nicht zu erkennen." (S. 13).

Die gleichberechtigte Teilhabe von Menschen mit hohem Unterstützungsbedarf im Bildungssystem und auf dem Arbeitsmarkt scheint noch lange auf sich warten zu lassen. 